\title{
Multiple Myeloma of the Central Nervous System: 13 Cases and Review of the Literature
}

\author{
Gergely Varga ${ }^{10},{ }^{1}$ Gábor Mikala, ${ }^{2}$ László Gopcsa, ${ }^{2}$ Zoltán Csukly, ${ }^{2}$ Sarolta Kollai, ${ }^{3}$ \\ György Balázs, ${ }^{4}$ Tímár Botond, ${ }^{5}$ Nikolett Wohner, ${ }^{1}$ Laura Horváth, ${ }^{1}$ Gergely Szombath, ${ }^{1}$ \\ Péter Farkas, ${ }^{1}$ and Tamás Masszi ${ }^{1}$ \\ ${ }^{1} 3 r d$ Department of Internal Medicine, Semmelweis University, Budapest, Hungary \\ ${ }^{2}$ Department of Haematology and Stem Cell Transplantation, St. István and St. László Hospital, Budapest, Hungary \\ ${ }^{3}$ Department of Neurology, Semmelweis University, Budapest, Hungary \\ ${ }^{4}$ Heart and Vascular Center, Division of Diagnostic Imaging, Semmelweis University, Budapest, Hungary \\ ${ }^{5} 1$ st Department of Pathology and Experimental Cancer Research, Semmelweis University, Budapest, Hungary \\ Correspondence should be addressed to Gergely Varga; vargager@gmail.com
}

Received 27 November 2017; Accepted 18 March 2018; Published 23 April 2018

Academic Editor: Minesh P. Mehta

Copyright (C) 2018 Gergely Varga et al. This is an open access article distributed under the Creative Commons Attribution License, which permits unrestricted use, distribution, and reproduction in any medium, provided the original work is properly cited.

\begin{abstract}
Central nervous system involvement is a rare complication of multiple myeloma with extremely poor prognosis as it usually fails to respond to therapy. We present 13 cases diagnosed at two centers in Budapest and review the current literature. The majority of our cases presented with high-risk features initially; two had plasma cell leukemia. Repeated genetic tests showed clonal evolution in 3 cases. Treatments varied according to the era, and efficacy was poor as generally reported in the literature. Only one patient is currently alive, with 3-month follow-up, and the patient responded to daratumumab-based treatment. Recent case reports show promising effectivity of pomalidomide and marizomib.
\end{abstract}

\section{Introduction}

Multiple myeloma (MM) typically presents with skeletal symptoms (back pain, vertebral fractures, and lytic lesions), cytopenias (anemia, thrombocytopenia, and neutropenia), renal failure, and infections related to immunoparesis [1]. Extramedullary propagation is a rare event with poor prognosis due to frequent treatment failure. Within this group, central nervous system (CNS) involvement is a particularly problematic complication with an even worse prognosis.

The treatment of MM developed a lot in recent years. From the early 2000s, first thalidomide and its analogues and then bortezomib and newer proteasome inhibitors were introduced, followed by anti-CD38 and anti-CS1 antibodies getting FDA and EMA approval most recently. As a result, the expected survival of MM increased to 5-10 years in the majority of patients [2]. The longer survival corresponds to more extensive disease evolution that sometimes results in previously unseen, more resistant, and more aggressive clones with features uncommon in historical cohorts. Normally myeloma cells need constant support from the bone marrow stromal cells; however, evolution of the disease sometimes leads to clones that can survive independently in extramedullary tissues. This is rare at presentation (7\%) but becomes increasingly common at subsequent relapses in the form of organ involvement, soft tissue tumors, plasma cell leukemia, and rarely (less than 1\%) CNS involvement, which is the focus of this paper [3]. Due to its rarity, clinical trials are missing in this field; therefore, most of our knowledge is derived from case reports and retrospective reviews. As a consequence, we do not have evidence-based treatment guidelines.

Based on a review by Dispenzieri and Kyle, intracranial plasmacytomas or myelomas can be classified into four groups: (1) those extending from the skull pressing inward, (2) those growing from the dura mater or the leptomeninges, (3) those arising from the mucous membranes of a nasopharyngeal plasmacytoma, and (4) intraparenchymal lesions without evidence of extension from any of these other three 


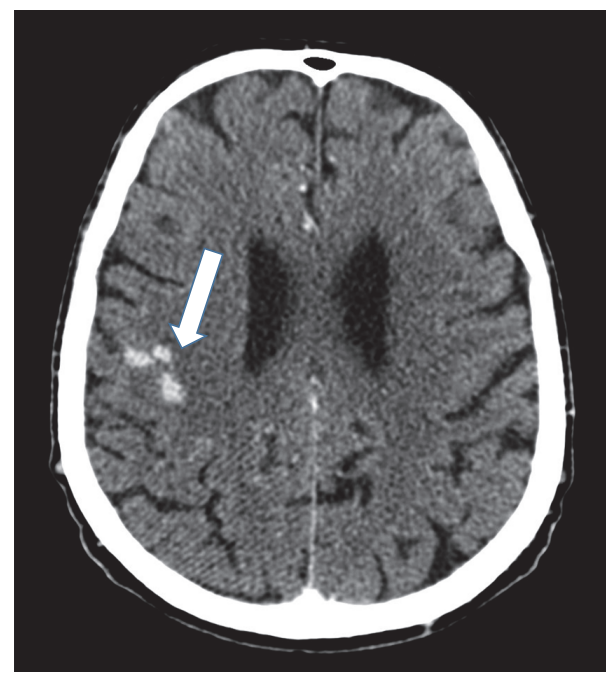

(a)

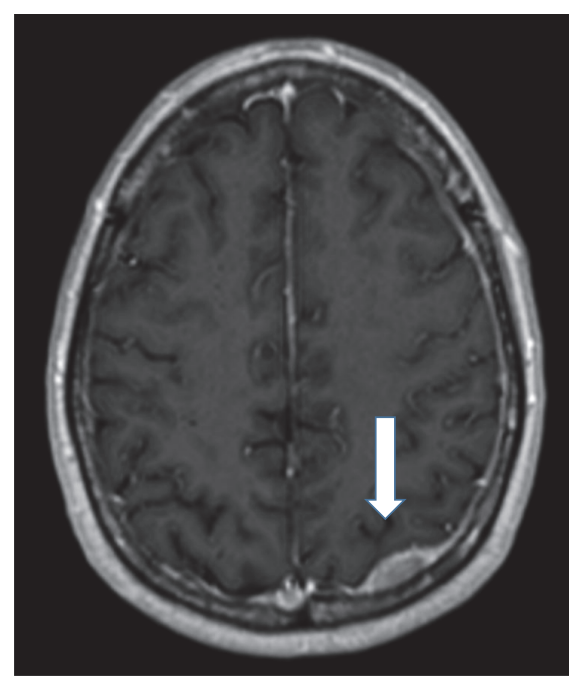

(b)

FIGURE 1: CT and MRI images of meningeal myeloma. (a) Contrast-enhanced CT; arrows: abnormally enhancing, leptomeningeal nodular lesions in the right frontal sulci (patient 4); (b) T1-weighted brain MRI after Gadolinium administration; arrows: abnormally enhancing dural lesion to be differentiated from subdural hemorrhage (patient 11).

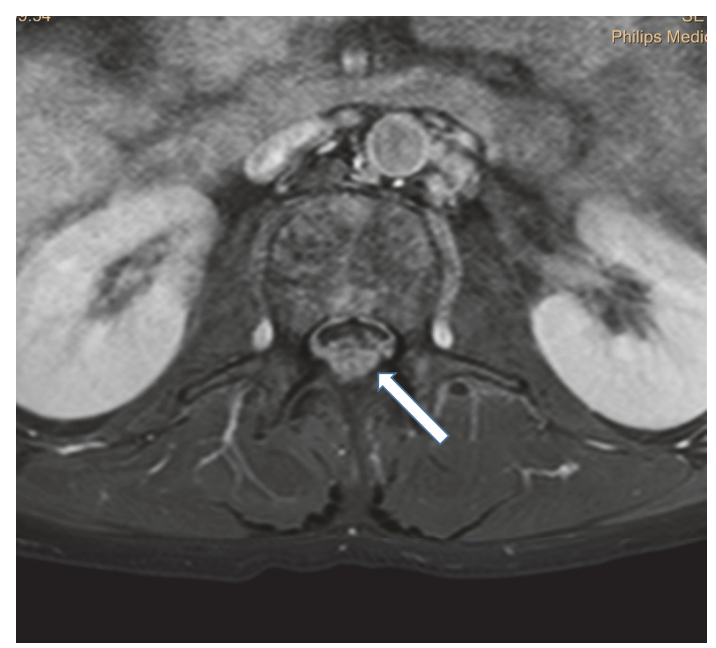

(a)

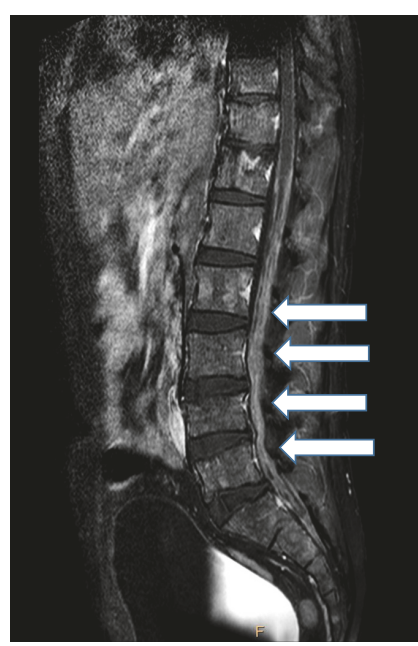

(b)

Figure 2: Gadolinium enhanced T1-weighted MRI images of leptomeningeal myeloma of the lumbosacral spine. (a) Axial and (b) sagittal image; arrows: contrast-enhanced myelomatous deposits (patient 11).

sites [4]. Leptomeningeal involvement is the most common form, often resulting in nerve root infiltration and cerebral nerve palsies.

\section{Symptoms}

Specific symptoms depend on the underlying abnormality. Headache, confusion, cerebral nerve palsy, and radiculopathy are the most common general symptoms at presentation. In case of intraparenchymal lesions, focal neurologic symptoms can sometimes occur together with somnolence or seizures. The diagnosis of CNS MM is based on imaging and/or cerebrospinal fluid (CSF) analysis, with stereotaxic brain biopsy, if applicable [5].

\section{Imaging}

In suspected CNS MM, the first diagnostic step is usually contrast-enhanced MRI (head and/or whole spine). The sensitivity of MRI is over 90\%: it can demonstrate bone-originated plasmacytomas, intraparenchymal tumorous lesions, or leptomeningeal contrast-enhanced deposits (Figures 1 and 2). In a review, the latter was the most common form, present in $70 \%$ of the cases [6]. CT can be diagnostic as well but with lower sensitivity (80\%), and iodinated contrast material is often contraindicated in myeloma [3]. However, the abnormalities are not necessarily specific; for example, they can mimic subdural hemorrhage as in patient 11 in our series [6] (Figure 1(b)). 


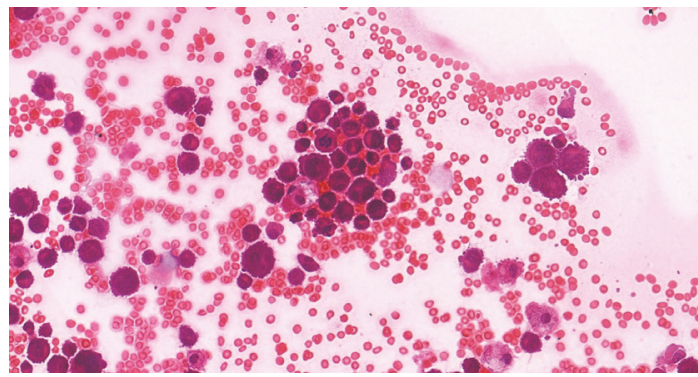

(a)

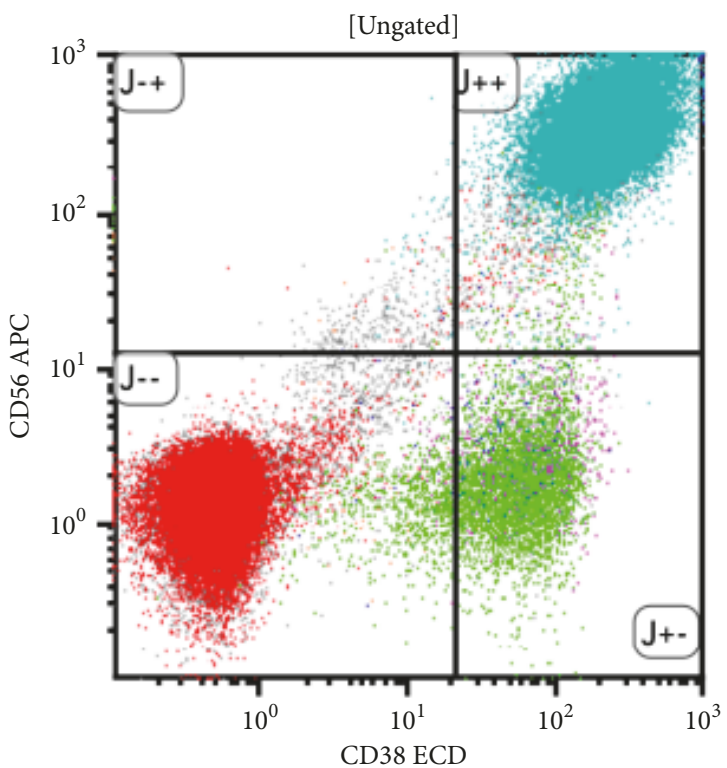

(c)

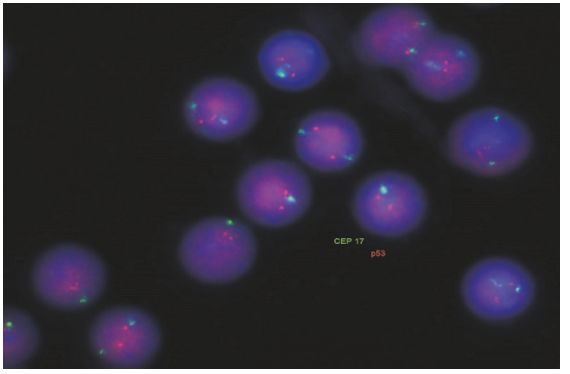

(b)

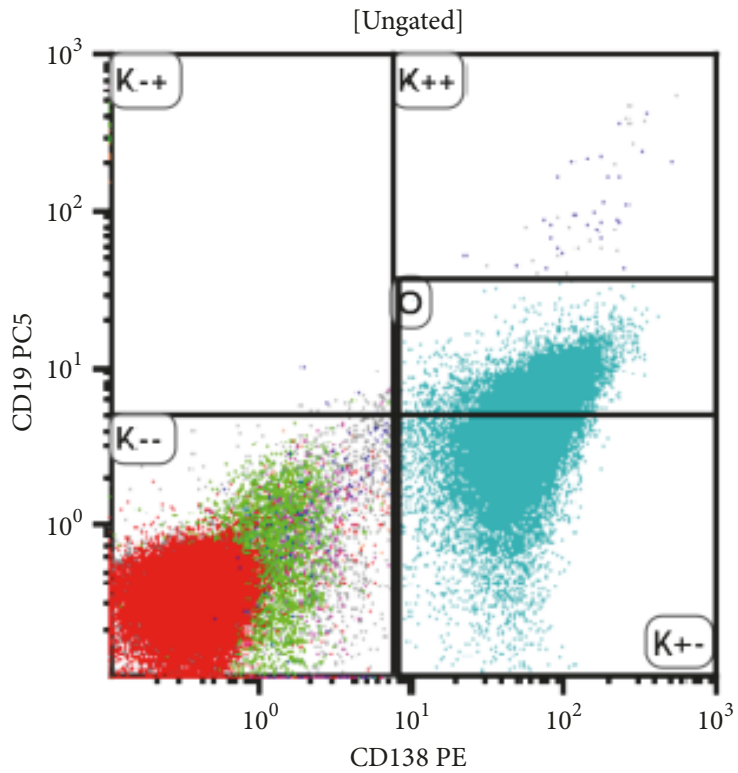

(d)

FIGURE 3: CSF analysis. (a) MGG stained cytospin preparation; (b) fluorescence in situ hybridization (FISH) with probes for 17p (normal); ((c) and (d)) flow cytometry: the plasma cells are CD38, 56, and 138 positive.

\section{CSF Analysis}

To cytologically confirm leptomeningeal disease, lumbar puncture is necessary: in the previously cited review, it was diagnostic in $90 \%$ of the cases [6]. Liquor opening pressure is typically raised, and the liquor cell count is elevated. Plasma cells can be cytologically identified (Figure 3(a)). Flow cytometry can confirm monoclonality and aberrant protein expression (Figures 3(c) and 3(d)). CSF protein electrophoresis and free light chain analysis can indirectly confirm the CNS presence of the aberrant plasma cell clone. Fluorescence in situ hybridization (FISH) on the cytospin preparation often shows high-risk features, most commonly deletion 17p (TP53) (Figure 3(b)) [12]. These genetic aberrations contribute to the resistance to traditional chemotherapy and radiotherapy [13].

\section{Differential Diagnosis}

Naturally, there can be many other causes for nervous system symptoms in myeloma patients, making the diagnosis of CNS propagation difficult. Many of the established MM drugs cause peripheral neuropathy. In case of bortezomib, this is usually dominantly sensory, while in case of thalidomide, it is typically a sensomotor neuropathy. Both cause axonal degeneration, and the symptoms are only reversible in case of quick drug interruption/dose reduction; however, if treatment continues, they can become permanent [14]. MM (and other M-protein secretory conditions from MGUS to Waldenström's macroglobulinemia) can cause polyneuropathy on their own right. M-protein is present in $10 \%$ of cryptogenic neuropathies, mostly IgM, and in $50 \%$ of the IgM cases the M-protein has an anti-myelin associated glycoprotein (anti-MAG) activity [15]. Another complication of the above-mentioned $\mathrm{M}$-protein producing diseases is $\mathrm{AL}$ amyloidosis. In case of clinical suspicion, tissue biopsy is needed to confirm the diagnosis of amyloid deposition. The most common biopsy targets are abdominal subcutaneous fat and either rectal mucosal or gingival biopsy.

Other complications, common in MM, such as hyperviscosity, renal failure, and hypercalcemia can also cause CNS symptoms, most commonly disorientation and somnolence. Given the average age of the MM population plus the prothrombotic properties of the IMiDs, vascular events (both ischemia and hemorrhage) can occur. Apart from 


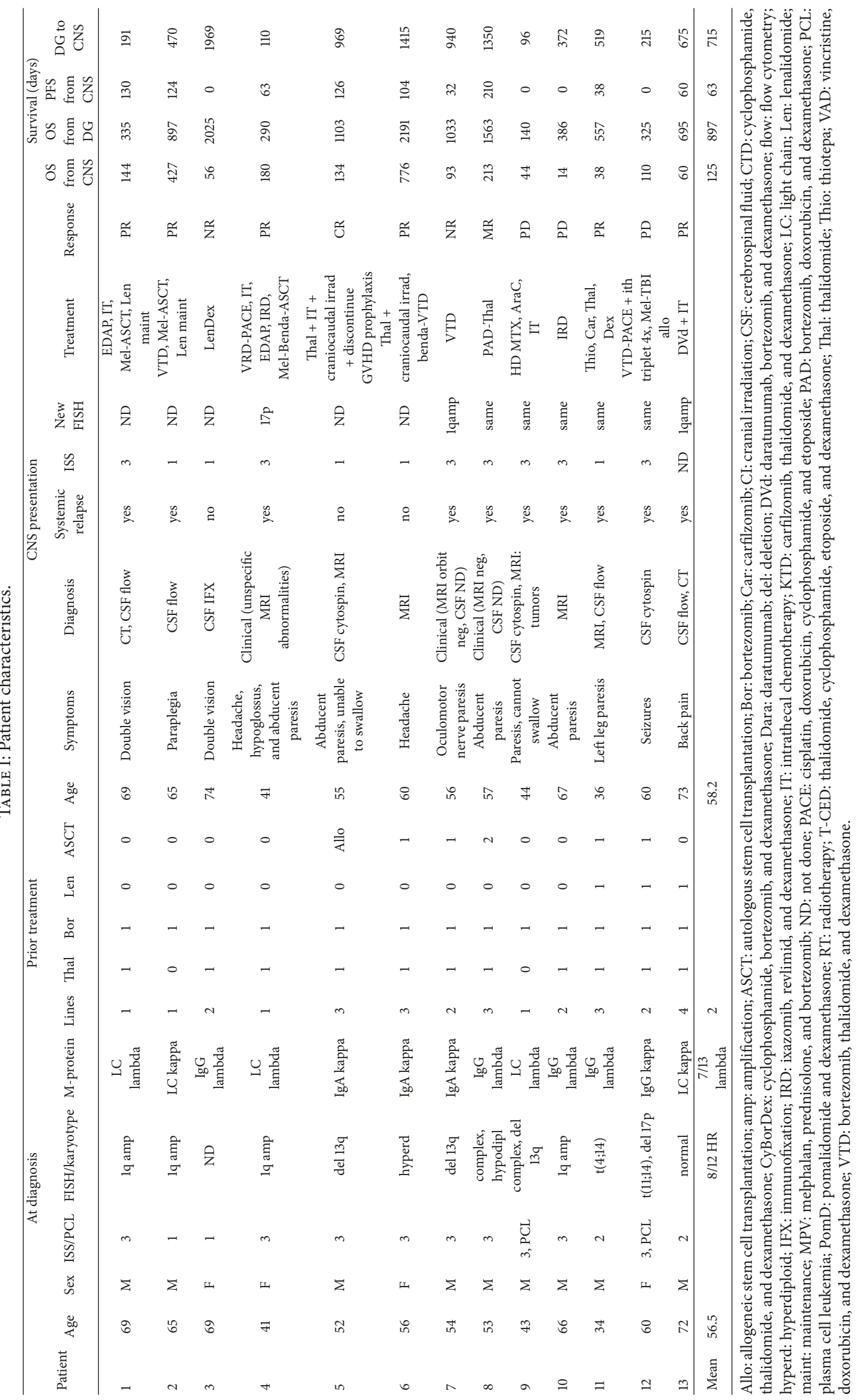


TABLE 2: Largest recently published case series.

\begin{tabular}{|c|c|c|c|c|c|}
\hline & $n$ & Era & $\begin{array}{l}\text { Median OS } \\
\text { (months, CI) }\end{array}$ & Center(s) & Reference \\
\hline Jurczyszyn et al. & 172 & 1995-2014 & 3.7 (NA) & Multicenter: 38 centers from 20 countries & {$[3]$} \\
\hline Paludo et al. & 29 & $1998-2014$ & $3.4(1-10)$ & Mayo Clinic, Rochester & {$[5]$} \\
\hline Schluterman et al. & 23 & $1990-2003$ & $3(0.1-25)$ & University of Arkansas, Little Rock & {$[6]$} \\
\hline Chen et al. & 37 & $1999-2010$ & $4.6(2.8-6.7)$ & Princess Margaret Cancer Centre, Toronto & [7] \\
\hline Abdallah et al. & 35 & $1996-2012$ & $4(1-13)$ & University of Arkansas, Little Rock & [8] \\
\hline Majd et al. & 9 & 1998-2012 & $3(1-12)$ & Mount Sinai Hospital, New York & [9] \\
\hline Gozzetti et al. & 50 & 2000-2010 & $6(1-23)$ & $\begin{array}{l}\text { GIMEMA (Gruppo Italiano Malattie EMatologiche } \\
\text { dell'Adulto) multiple myeloma working party }\end{array}$ & {$[10]$} \\
\hline Dias et al. & 21 & 2008-2016 & $5.8(\mathrm{NA})$ & Faculdade de Ciências Médicas da Santa Casa de São Paulo & {$[11]$} \\
\hline
\end{tabular}

CI: confidence interval; NA: not available.

these, autoimmunity, paraneoplasia, and infections have to be considered (herpes and JC viruses) in these patients with compromised immune system.

Most myeloma patients are taking multiple medications. Among these, thalidomide has a sedative effect, but patients taking thalidomide often report vertigo, tremor, imbalance, and difficulty to walk too. These tend to improve quickly upon stopping thalidomide. Major analgesics (morphine and fentanyl) and adjuvant pain medications (gabapentin, pregabalin, tegretol, and tricyclic antidepressants) can blur the clinical picture further, especially if drug levels are unreliable due to liver and kidney failure.

\section{Treatment and Survival}

The treatment of CNS MM is highly problematic. On one hand, this complication is more common in otherwise highrisk MM (both clinically aggressive and genetically high risk), when chemotherapy is usually less effective. Another problem is that drugs tested and proven in relapsed MM, such as the proteasome inhibitors (bortezomib, carfilzomib, and ixazomib), cannot cross the blood-brain barrier, and standard chemotherapy drugs used to treat CNS lymphoma and brain cancer are not particularly effective in MM. The IMiDs (thalidomide, lenalidomide, and pomalidomide) do cross the blood-brain barrier; however, IMiD resistance is not uncommon in this group of patients. The anti-CD38 antibody daratumumab and anti-CS1 elotuzumab recently approved in relapsed MM were not prospectively tested in this particular group of patients; CNS involvement was an exclusion criterion in the registration trials. It is unknown whether these antibodies can get through the blood-brain barrier, and there is no published report about their intrathecal use, whatever tempting it would be theoretically. In one case report of a CNS MM case, it was found that MM cells in the CSF shred their surface CD38 after starting systemic daratumumab, which was interpreted as an indirect sign of CNS penetration of the drug [16]. It has to be mentioned here that intrathecal injection of bortezomib can be fatal and is therefore absolutely contraindicated!

As a salvage, in analogy to acute lymphoid leukemia, intrathecal combinational chemotherapy with methotrexate, cytosine arabinoside, and dexamethasone (15 mg, $40 \mathrm{mg}$, and $4 \mathrm{mg}$, resp.) is often applied continuously until liquor clearance, usually in combination with high-dose chemotherapy, using drugs with known CNS penetration (methotrexate, cytosine arabinoside, idarubicin, and thiotepa), together with high-dose dexamethasone, similar to what we normally do in CNS lymphoma. In case of a response to this strategy, retrospective data support the use of craniocaudal radiotherapy and possibly ASCT as consolidation [7].

Recently, a case review reported the effectivity of pomalidomide in CNS MM [17]. Pomalidomide is a drug of the IMiD class approved for lenalidomide refractory patients, which according to a subgroup analysis is more effective in high-risk cytogenetics, especially deletion $17 \mathrm{p}$, than other IMiDs and has good CNS penetration [18]. Another drug that could have a role in this setting is marizomib, a potent natural second-generation proteasome inhibitor produced by a marine bacterium, which was used successfully in two cases of CNS MM [16].

Based on these data, it is not surprising that the overall survival in this condition is extremely poor. The survival data varies widely between studies but usually does not exceed a couple of months from diagnosis (median survival: $2-8$ months, Table 2) [3,5-11, 19]. It is significantly better, however, in those patients who had craniocaudal irradiation or ASCT. Although this data is biased as these are selected patients who responded to their initial treatment $[3,5]$, in more recent publications, nevertheless, there is a promising increase in survival [7].

\section{13 Cases of CNS MM over 10 Years in Our 2 Centers}

Out of $548 \mathrm{MM}$ cases treated over 10 years at the 3rd Department of Internal Medicine, Semmelweis University, and the Department of Haematology and the Stem Cell Transplantation, St. István and St. László Hospital, we identified retrospectively 13 cases of CNS MM. Patients' characteristics including symptoms, treatments, and survival are shown in Table 1, treatment courses and responses to various treatment lines are depicted graphically in Figure 4, and progressionfree survival and overall survival given in Figure 5. 


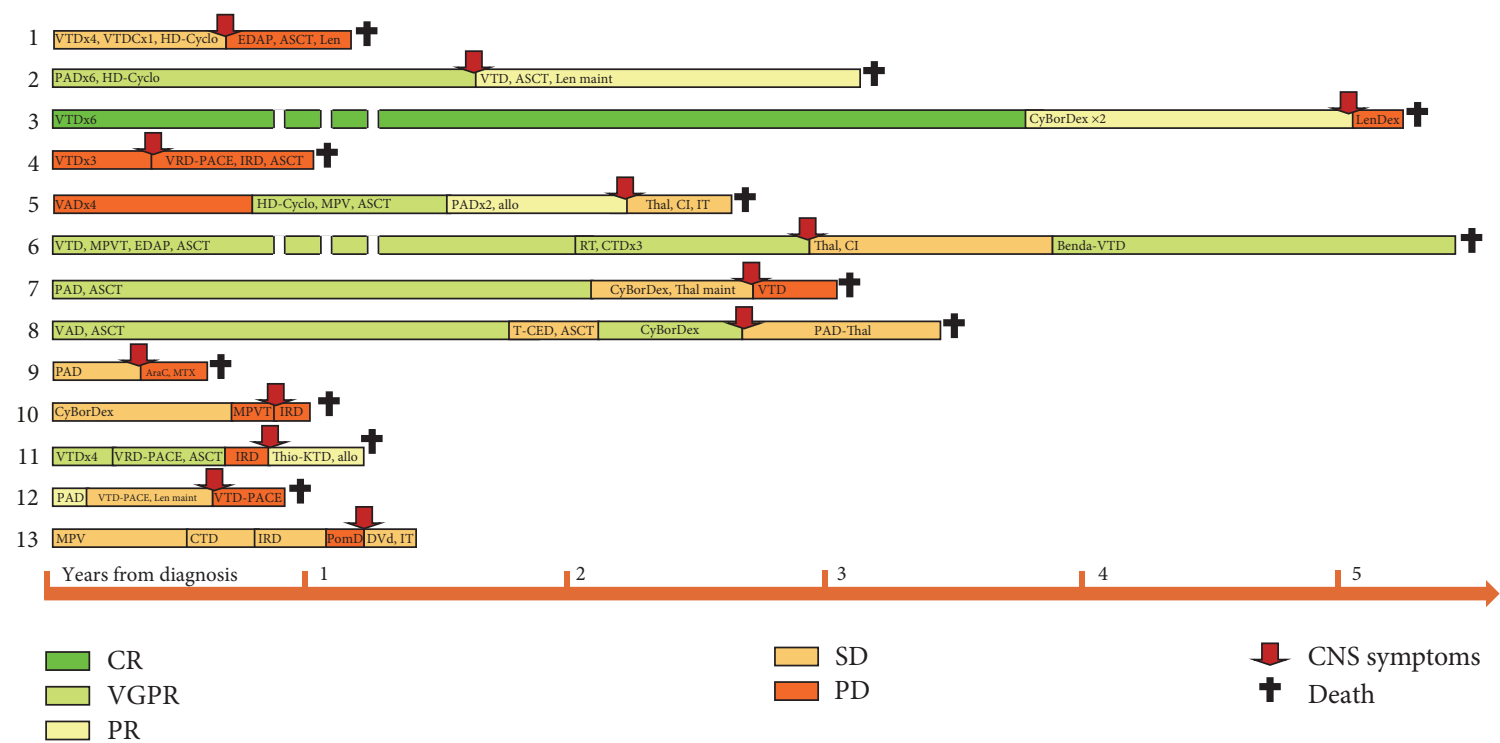

Figure 4: Treatments and responses. Allo: allogeneic stem cell transplantation; amp: amplification; ASCT: autologous stem cell transplantation; Bor: bortezomib; Car: carfilzomib; CI: cranial irradiation; CSF: cerebrospinal fluid; CTD: cyclophosphamide, thalidomide, and dexamethasone; CyBorDex: cyclophosphamide, bortezomib, and dexamethasone; Dara: daratumumab; del: deletion; DVd: daratumumab, bortezomib, and dexamethasone; flow: flow cytometry; hyperd: hyperdiploid; IFX: immunofixation; IRD: ixazomib, revlimid, and dexamethasone; IT: intrathecal chemotherapy; KTD: carfilzomib, thalidomide, and dexamethasone; LC: light chain; Len: lenalidomide; maint: maintenance; MPV: melphalan, prednisolone, and bortezomib; ND: not done; PACE: cisplatin, doxorubicin, cyclophosphamide, and etoposide; PAD: bortezomib, doxorubicin, and dexamethasone; PCL: plasma cell leukemia; PomD: pomalidomide and dexamethasone; RT: radiotherapy; T-CED: thalidomide, cyclophosphamide, etoposide, and dexamethasone; Thal: thalidomide; Thio: thiotepa; VAD: vincristine, doxorubicin, and dexamethasone; VRD: bortezomib, lenalidomide, and dexamethasone; VTD: bortezomib, thalidomide, and dexamethasone.

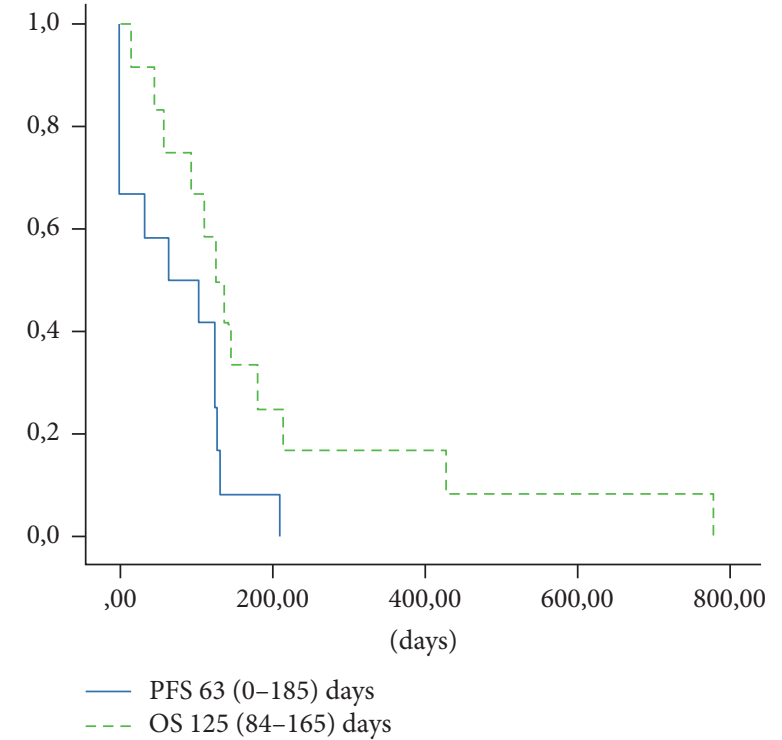

FIGURE 5: Progression-free survival (PFS) and overall survival (OS).

CNS symptoms started in each case at the time of relapse, on average 2 years from the initial diagnosis of MM (range: 3 months -5.3 years). These patients were younger than the usual MM patients, with more IgA and LC secretory cases than usually seen in MM. In 8/13 cases, high-risk cytogenetics were present at diagnosis, and we observed clonal evolution in terms of new FISH findings in 3 patients. Two patients presented originally as primary plasma cell leukemia; in these cases, survival from the original diagnoses was only 5 and 12 months.

The dominant symptoms were cranial nerve palsies (double vision and dysphagia) but patients also presented with headache, seizures, paraparesis or paraplegia, and radiculopathy. The diagnosis was supported by imaging in 6 and by CSF analysis in 8 cases.

Treatment strategies varied according to the patients' presenting symptoms, performance stage, and drug availability at the time. Ten patients had treatment containing an IMiD (5 lenalidomide and 5 thalidomide), five had high-dose salvage chemotherapy, four ASCT, two craniocaudal irradiation, and six intrathecal chemotherapy. One very recent case (\#13) presenting with CNS relapse in September 2017 showed a remarkable response to systemic daratumumab. Daratumumab was combined with bortezomib and high-dose corticosteroids and the patient had 3 times intrathecal triplet chemotherapy treatment as well. With 3-month follow-up, he is alive and well, and his CSF that was previously full of plasma cells (Figure 3(a)) is now clear.

In one case, CNS MM presented as an isolated relapse following allogeneic SCT, indicating that graft versus myeloma effect is less prominent in the CNS. In another case, after combination chemotherapy for CNS MM, we attempted allogeneic SCT with total body irradiation (TBI) based conditioning; this patient died in sepsis but was apparently free of disease. 
Survival, in keeping with the literature, was very poor in our series; with the median being 3 months, the longest survival so far was 14 months.

\section{Concluding Remarks}

As in the 13 cases presented in our series, CNS MM is a real challenge to the treating physician. The diagnosis is complex; probably many cases remain undetected. Although there are no clear guidelines regarding best treatment, aggressive management is necessary. Most recently, pomalidomide and marizomib showed promising results, and our cases suggest that monoclonal antibodies, particularly daratumumab, might have a role in this setting, too.

\section{Conflicts of Interest}

The authors have no conflicts of interest.

\section{References}

[1] S. V. Rajkumar, M. A. Dimopoulos, and A. Palumbo, "International Myeloma Working Group updated criteria for the diagnosis of multiple myeloma," The Lancet Oncology, vol. 15, no. 12, pp. e538-e548, 2014.

[2] S. K. Kumar, A. Dispenzieri, M. Q. Lacy et al., "Continued improvement in survival in multiple myeloma: Changes in early mortality and outcomes in older patients," Leukemia, vol. 28, no. 5, pp. 1122-1128, 2014.

[3] A. Jurczyszyn, N. Grzasko, A. Gozzetti et al., "Central nervous system involvement by multiple myeloma: A multi-institutional retrospective study of 172 patients in daily clinical practice," American Journal of Hematology, vol. 91, no. 6, pp. 575-580, 2016.

[4] A. Dispenzieri and R. A. Kyle, "Neurological aspects of multiple myeloma and related disorders," Best Practice \& Research Clinical Haematology, vol. 18, no. 4, pp. 673-688, 2005.

[5] J. Paludo, U. Painuly, and S. Kumar S, "Myelomatous involvement of the central nervous system , Clinical Lymphoma," Myeloma \& Leukemia, vol. 16, no. 11, pp. 644-654, 2016.

[6] K. O. Schluterman, A. B.-T. Fassas, R. L. Van Hemert, and S. I. Harik, "Multiple myeloma invasion of the central nervous system," JAMA Neurology, vol. 61, no. 9, pp. 1423-1429, 2004.

[7] C. I. Chen, E. Masih-Khan, H. Jiang et al., "Central nervous system involvement with multiple myeloma: long term survival can be achieved with radiation, intrathecal chemotherapy, and immunomodulatory agents," British Journal of Haematology, vol. 162, no. 4, pp. 483-488, 2013.

[8] A. O. Abdallah, S. Atrash, Z. Shahid, and etal., "Patterns of central nervous system involvement in relapsed and refractory multiple myeloma," Clinical Lymphoma, Myeloma \& Leukemia, vol. 14, no. 3, pp. 211-214, 2014.

[9] N. Majd, A. Demopoulos, and A. Chari, "Central nervous system involvement in multiple myeloma patients in the era of novel therapies, neurology," Neurology, vol. 80, no. 7, 2013.

[10] A. Gozzetti, A. Cerase, F. Lotti et al., "Extramedullary intracranial localization of multiple myeloma and treatment with novel agents: a retrospective survey of 50 patients," Cancer, vol. 118, no. 6, pp. 1574-1584, 2012.
[11] A. L. Dias, F. Higashi, A. L. Peres, P. Cury, E. d. Crusoé, and V. T. Hungria, "Multiple myeloma and central nervous system involvement: experience of a Brazilian center," Hematology, Transfusion and Cell Therapy, vol. 40, no. 1, pp. 30-36, 2018.

[12] H. Chang, S. Sloan, D. Li, and A. K. Stewart, "Multiple myeloma involving central nervous system: high frequency of chromosome 17p13.1 (p53) deletions," British Journal of Haematology, vol. 127, no. 3, pp. 280-284, 2004.

[13] A. B.-T. Fassas, F. Muwalla, T. Berryman et al., "Myeloma of the central nervous system: association with high-risk chromosomal abnormalities, plasmablastic morphology and extramedullary manifestations," British Journal of Haematology, vol. 117, no. 1, pp. 103-108, 2002.

[14] I. Istenes, Zs. Nagy, and J. Demeter, "Chemotherapy-induced peripheral neuropathy: characteristics, diagnosis and treatment," Hungarian Oncology, vol. 60, pp. 165-175, 2016.

[15] R. A. Rison and S. R. Beydoun, "Paraproteinemic neuropathy: A practical review," BMC Neurology, vol. 16, no. 1, article no. 13, 2016.

[16] A. Badros, Z. Singh, B. Dhakal et al., "Marizomib for central nervous system-multiple myeloma," British Journal of Haematology, vol. 177, no. 2, pp. 221-225, 2017.

[17] A. Mussetti, S. Dalto, and V. Montefusco, "Effective treatment of pomalidomide in central nervous system myelomatosis," Leukemia \& Lymphoma, vol. 54, no. 4, pp. 864-866, 2013.

[18] X. Leleu, L. Karlin, M. Macro et al., "Pomalidomide plus lowdose dexamethasone in multiple myeloma with deletion $17 \mathrm{p}$ and/or translocation (4;14): IFM 2010-02 trial results," Blood, vol. 125, no. 9, pp. 1411-1417, 2015.

[19] L. Nieuwenhuizen and D. H. Biesma, "Central nervous system myelomatosis: review of the literature," European Journal of Haematology, vol. 80, no. 1, pp. 1-9, 2008. 


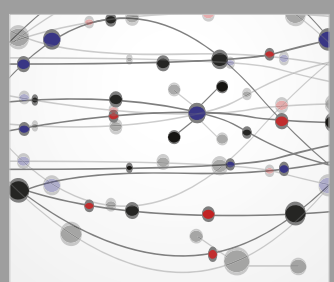

The Scientific World Journal
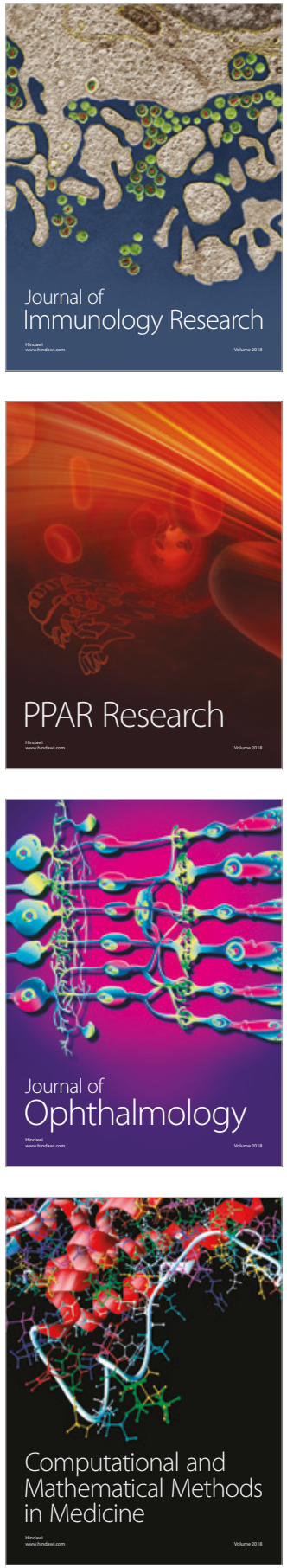

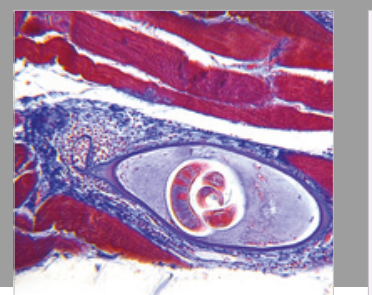

Gastroenterology Research and Practice

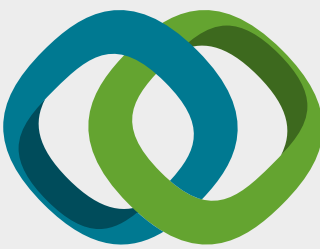

\section{Hindawi}

Submit your manuscripts at

www.hindawi.com
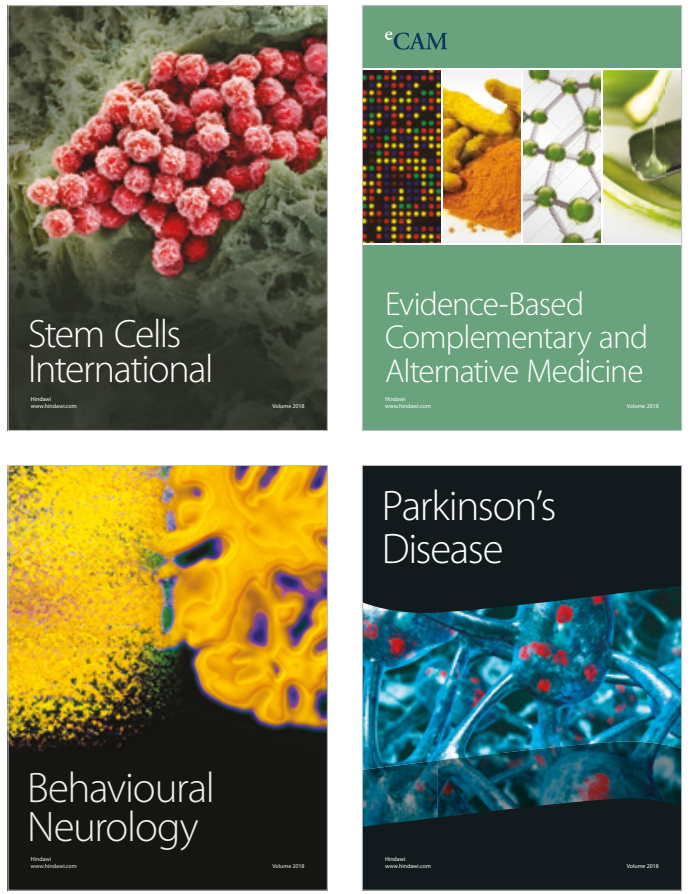

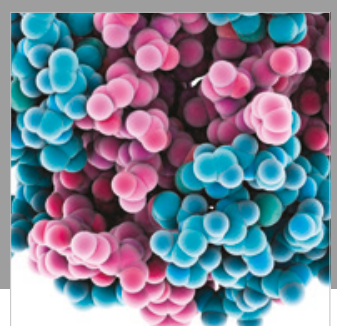

ournal of

Diabetes Research

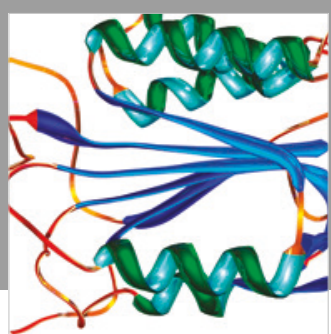

Disease Markers
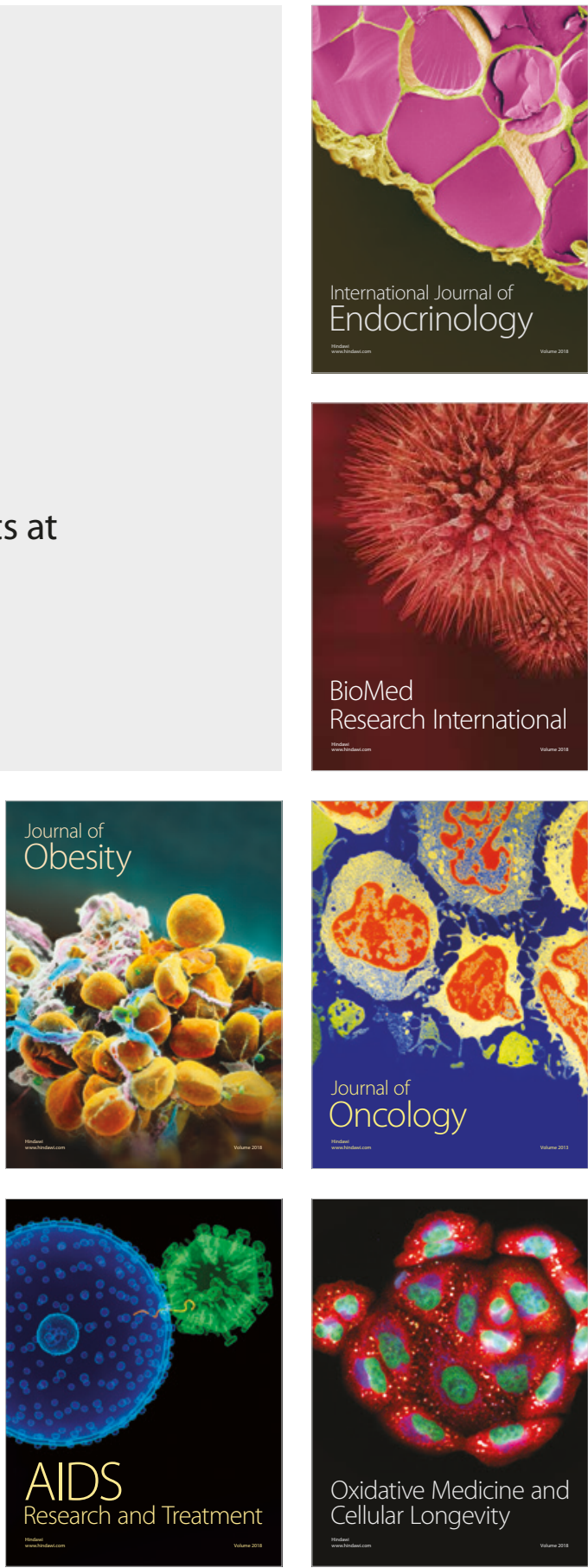